\title{
The aqueous extract of Gentianella acuta improves isoproterenol-induced myocardial fibrosis via inhibition of the TGF-ß1/Smads signaling pathway
}

\author{
HONG-XIA YANG ${ }^{1}$, GENG-RUI XU ${ }^{1}$, CHUANG ZHANG $^{1}$, JIA-HUAN SUN ${ }^{2}$, YUE ZHANG ${ }^{1,3}$, \\ JUN-NA SONG ${ }^{4}$, YUN-FENG LI ${ }^{1,3}$, YU LIU ${ }^{1,3}$ and AI-YING LI ${ }^{1,3}$
}

\begin{abstract}
${ }^{1}$ Department of Biochemistry and Molecular Biology, College of Basic Medicine, Hebei University of Chinese Medicine;
${ }^{2}$ Department of Medical Laboratory Science, College of Integration of Chinese and Western Medicine, Hebei University of Chinese Medicine, Shijiazhuang, Hebei 050200; ${ }^{3}$ Hebei Key Laboratory of Chinese Medicine Research on Cardio-cerebrovascular Disease, Shijiazhuang, Hebei 050091; ${ }^{4}$ Department of Medicinal Plant, College of Pharmacy, Hebei University of Chinese Medicine, Shijiazhuang, Hebei 050200, P.R. China
\end{abstract}

Received July 20, 2019; Accepted October 31, 2019

DOI: $10.3892 /$ ijmm.2019.4410

\begin{abstract}
Gentianella acuta (G. acuta) is one of the most commonly used herbs in Chinese Mongolian medicine for the treatment of heart disease. Previously, it was found that G. acuta ameliorated cardiac function and inhibited isoproterenol (ISO)-induced myocardial fibrosis in rats. In this study, the underlying anti-fibrotic mechanism of G. acuta was further elucidated. Histopathological changes in the heart were observed by hematoxylin-eosin, Masson trichrome and wheat germ agglutinin staining. Relevant molecular events were investigated using immunohistochemistry and western blotting. The results revealed that G. acuta caused improvements in myocardial injury and fibrosis. G. acuta also inhibited collagens I and III and $\alpha$-smooth muscle actin production in heart tissue. G. acuta downregulated the expression of transforming growth factor $\beta 1$ (TGF- $\beta 1$ ) and notably inhibited the levels of phosphorylation of TGF- $\beta$ receptors I and II. Furthermore, G. acuta caused downregulation of the intracellular mothers against decapentaplegic homolog (Smads) 2 and 4 expression and inhibited Smads 2 and 3 phosphorylation. The results further demonstrated that the mechanism underlying anti-myocardial fibrosis effects of G. acuta was based upon the suppression of the TGF- $\beta 1 /$ Smads signaling pathway. Therefore, G. acuta may be a potential therapeutic agent for ameliorating myocardial fibrosis.
\end{abstract}

Correspondence to: Dr Ai-Ying Li, Department of Biochemistry and Molecular Biology, College of Basic Medicine, Hebei University of Chinese Medicine, 3 Xing Yuan Road, Luquan, Shijiazhuang, Hebei 050200, P.R. China

E-mail: liaiying@hebcm.edu.cn

Key words: Gentianella acuta, isoproterenol, myocardial fibrosis, transforming growth factor- $\beta 1$, transforming growth factor- $\beta 1 /$ mothers against decapentaplegic homolog signaling pathway

\section{Introduction}

Myocardial fibrosis, which is a morphological feature of ventricular remodeling, develops as a result of several different heart diseases, including myocardial infarction, hypertension, atherosclerosis and myocarditis $(1,2)$. Excessive extracellular matrix (ECM) production often disrupts the tissues' physiological architecture (3). In the heart, the primary components of the ECM network structure are predominantly collagens I and III. Massive cardiac collagen deposition can lead to ventricular stiffness and diastolic and systolic dysfunction $(4,5)$. These adverse changes can result in heart failure and even sudden cardiac death $(4,6,7)$. Treatment strategies for myocardial fibrosis are still a focus of clinical attention.

Transforming growth factor $\beta 1$ (TGF- $\beta 1$ ) has been confirmed as a primary regulator of myocardial fibrosis $(8,9)$. TGF- $\beta 1$ exerts its biological functions by binding to the active TGF- $\beta$ receptors type I and II (T $\beta$ RI and II), which causes phosphorylation of T $\beta$ RI and II (9). The activated receptors subsequently phosphorylate receptor-regulated mothers against decapentaplegic homolog (Smad) proteins and increase the common-regulated Smad protein expression. These Smad proteins are the primary downstream signaling molecules of TGF- $\beta 1$ (10). It is well known that the upregulated TGF- $\beta$ expression resulting from tissue injury plays a role in the tissue repair process and scar formation (11). In terms of post-infarct remodeling, TGF- $\beta 1$ is involved in myocardial fibrosis via ECM synthesis upregulation by fibroblasts; as a consequence, TGF- $\beta 1 /$ Smads is considered to be the primary signaling pathway that contributes to myocardial fibrosis (12). Therefore, the inhibition of TGF- $\beta$ signaling is a promising approach for inhibiting myocardial fibrosis.

Gentianella acuta (G. acuta), which belongs to the Gentianaceae, is one of the most commonly used herbs in Mongolian medicine to cure hepatitis, jaundice, fever and headache. In China, this herb is widely distributed in Hebei, Inner Mongolia, Shanxi and Shandon. The whole grass of G. acuta has been used to treat angina by the local herdsmen in Inner 
Mongolia for a long time. In these regions, G. acuta was called 'guixincao'. Ewenki people had a habit of drinking 'guixincao' tea and used it to treat angina by chewing or water decoction (13). Certain experimental results have indicated that $G$. acuta exerts anti-inflammatory, antioxidant, antibacterial and antiarrhythmic effects, thereby preventing cardiovascular and cerebrovascular diseases (14-16). Numerous studies have shown that G. acuta and its active components have a protective effect on the heart $(16,17)$. In the authors' previous study, it was found that G. acuta significantly improved cardiac function and inhibited myocardial fibrosis in a rat model (18). However, the detailed mechanism underlying G. acuta's anti-myocardial fibrotic effects is not clear. The aim of the present study was to investigate whether the mechanism underlying G. acuta's anti-myocardial fibrosis effects is based on the TGF- $\beta 1 /$ Smads signaling pathway, which will provide evidence for clinical application.

\section{Materials and methods}

Primary drugs and reagents. Isoproterenol (ISO) was purchased from Tokyo Chemical Industry, Ltd. Bellidifolin, swertianolin and demethylbellidifolin were acquired from Chengdu Alfa Biotechnology, Co., Ltd. Masson trichrome and wheat germ agglutinin (WGA) staining kit were purchased from Wuhan Servicebio Technology, Ltd. Antibodies for $\alpha$-smooth actin ( $\alpha$-SMA; cat. no. ab32575), TGF- $\beta 1$ (cat. no. ab92486), Smad4 (cat. no. ab40759), phosphorylated (P)- and total Smads2 (cat. nos. ab53100 and ab40855) and 3 (cat. nos. ab52903 and ab40854) were purchased from Abcam. The antibody for T $\beta$ RI (cat. no. GB11271) was purchased from Wuhan Servicebio Technology, Ltd. The antibody for T $\beta$ RII (cat. no. A11788) was purchased from ABclonal Biotech Co., Ltd. Antibodies for collagens I (cat. no. AF7001), III (cat. no. AF0136), phospho-T $\beta$ RI (cat. no. AF8080) and II (cat. no. AF8191) were purchased from Affinity Biosciences. Antibody for GAPDH (cat. no. 60004-1-Ig) was obtained from Wuhan Sanying Biotechnology. Horseradish peroxidase (HRP)-conjugated anti-rabbit IgG (cat. no. ZDR-5306) and HRP-conjugated anti-mouse IgG (cat. no. ZDR-5307) antibodies were obtained from Beijing Zhongshan Golden Bridge Biotechnology, Co., Ltd.; OriGene Technologies, Inc. The SP9000 immunohistochemical staining kit was purchased from Beijing Zhongshan Golden Bridge Biotechnology, Co., Ltd.; OriGene Technologies, Inc.

Preparation of plant material. The whole plant of G. acuta was purchased from the market in Inner Mongolia and authenticated by Professor Zheng Yu-Guang (Department of Pharmacognosy, School of Pharmacy, Hebei University of Chinese Medicine). According to body surface area conversion equations (human: Rat=1: 6.3) for translating dosages from human to animal $(19,20)$, the clinical dosage of $G$. acuta $(6 \mathrm{~g} / \mathrm{d})$ in adults is $\sim$ equal to the daily dosage of $0.6 \mathrm{~g} / \mathrm{kg}$ in rat. The dosages of G. acuta adopted in the experiments are 0.3 , 0.6 and $1.2 \mathrm{~g} / \mathrm{kg}$, respectively. G. acuta's dried whole plant was ground and soaked in a 20 -fold volume of distilled water for $20 \mathrm{~min}$. The mixture, which was decocted twice at $100^{\circ} \mathrm{C}$ for $30 \mathrm{~min}$, was then filtered and concentrated at $80^{\circ} \mathrm{C}$. The dark brown aqueous extracts were stored in a refrigerator at $4^{\circ} \mathrm{C}$ until use.
Animal protocol. A total of 50 male Sprague-Dawley rats (5 weeks old) were obtained from the Experimental Animal Centre of Hebei Medical University (Shijiazhuang, China) and maintained in an animal facility $\left(22-23^{\circ} \mathrm{C} ; 55-60 \%\right.$ humidity) on a 12-h light/ dark cycle with free access to food and water. All experiments were conducted in accordance with the P.R. China legislation for the use and care of laboratory animals. The animal care and study protocols were approved by the Institutional Animal Care and Use Committee of Hebei University of Chinese Medicine (no. 1703440).

The myocardial fibrosis model was established as described previously $(21,22)$. Briefly, ISO $(5 \mathrm{mg} / \mathrm{kg} /$ day $)$ was injected subcutaneously into rats for seven days. A total of 50 rats were equally divided into the following five groups at random: i) Control group (Control); ii) model group (ISO); iii) low-dose drug intervention group (ISO+G. acuta $0.3 \mathrm{~g} / \mathrm{kg}$ ); iv) middle-dose drug intervention group (ISO+G. acuta $0.6 \mathrm{~g} / \mathrm{kg}$ ); and v) high-dose drug intervention group (ISO+G. acuta $1.2 \mathrm{~g} / \mathrm{kg}$ ). All drugs were administered via oral gavage and the treatment period lasted 21 days beginning from the day after the first ISO injection. On the 22nd day, the rats were anaesthetized and then sacrificed. All the samples were cut from the same location of heart, the mid left ventricular ( $2.5 \mathrm{~mm}$ above the apex). The tissues were then fixed for $24 \mathrm{~h}$ at room temperature with $4 \%$ paraformaldehyde for histopathological analyses and immunohistochemical measurements, and the fresh myocardial tissues were stored at $-80^{\circ} \mathrm{C}$ for western blotting analysis.

Morphological detection of heart. The heart tissues were fixed in $4 \%$ paraformaldehyde, embedded in paraffin wax and cut into $5-\mu \mathrm{m}$ sections. Hematoxylin and eosin (H\&E), Masson trichrome and WGA staining were performed in accordance with the manufacturer's protocol in order to observe histopathological changes and collagen deposition in the heart tissues. Sections were dehydrated, made transparent and sealed. Staining was visualized and images were captured using light microscopy and fluorescence microscope (Leica Microsystems $\mathrm{GmbH}$ ).

Immunohistochemical staining. Immunohistochemical staining was performed for detection of collagens I and III, $\alpha$-smooth muscle actin ( $\alpha$-SMA), TGF- $\beta 1$, Smads 2,3 and 4 expression according to the manufacturer's protocol of the SP9000 immunohistochemical staining kit. Briefly, heart samples were fixed in $4 \%$ paraformaldehyde, embedded in paraffin and cut into $5-\mu \mathrm{m}$ sections. The sections were then de-paraffinized, incubated with $3 \%$ hydrogen peroxide for $10 \mathrm{~min}$ at room temperature in order to block endogenous peroxidase activity and then incubated for $30 \mathrm{~min}$ at room temperature with $3 \%$ goat serum (OriGene Technologies, Inc.) to block nonspecific binding. Next, the sections were incubated with a primary antibody (collagen I, 1:100; collagen III, 1:100; $\alpha$-SMA, 1:500; TGF- $\beta 1,1: 50$; Smad2, 1:50; Smad3, 1:1,000; and Smad4, 1:100) at $4^{\circ} \mathrm{C}$ overnight. After washing with phosphate-buffered saline (PBS), the sections were incubated with secondary antibodies from the SP9000 kit for $15 \mathrm{~min}$ each at room temperature. Finally, the sections were incubated with 3, 3'-diaminobenzedine for $2 \mathrm{~min}$ at room temperature and observed under a light microscope (Leica Microsystems $\mathrm{GmbH}$ ). 
Western blotting. The heart tissues were washed twice with ice-cold PBS and lysed in radioimmunoprecipitation assay buffer containing a protease inhibitor cocktail, phenylmethyl sulfonyl fluoride, and phosphatase inhibitors. The protein concentration was measured using a bicinchoninic acid Protein Assay kit (Wuhan Servicebio Technology, Ltd.). An equal amount of protein $(20 \mu \mathrm{g} / \mathrm{lane})$ was separated using $10 \%$ SDS-PAGE and then transferred to polyvinylidene fluoride membranes. The membranes were blocked with $5 \%$ skim milk for $90 \mathrm{~min}$ at room temperature and then individually incubated with the appropriate primary antibody (collagen I, $1: 1,000$; collagen III, $1: 1,000 ; \alpha-$ SMA, $1: 5,000 ;$ TGF- $\beta 1$, 1:1,000; T $\beta$ RI, 1:1,000; P-T $\beta$ RI, 1:3,000; T $\beta$ RII, 1:1,000; P-TßRII, 1:3,000; Smad2, 1:2,000; P-Smad2, 1:1,000; Smad3, 1:10,000; P-Smad3, 1:2,000; and Smad4, 1:5,000) at $4^{\circ} \mathrm{C}$ overnight. Subsequently, the membranes were incubated with the appropriate HRP-conjugated secondary antibody (HRP-conjugated anti-rabbit IgG and HRP-conjugated anti-mouse $\mathrm{IgG}$, both 1:20,000) at room temperature for $60 \mathrm{~min}$. The immunoreactive bands were detected using the enhanced luminol-based ECL reagent kit (Invitrogen; Thermo Fisher Scientific, Inc.) and the Fusion FX5 Spectra multifunction laser-scanning system. The expression of each protein of interest was normalized to that of GAPDH and was analyzed using Image J 3.0 software (National Institute of Health).

High performance liquid chromatography (HPLC) analysis. The sample solutions were prepared by dissolving $0.2 \mathrm{~g}$ of G. acuta in $50 \mathrm{ml}$ water. The aqueous extracts of $G$. acuta were evaluated by HPLC analysis. The standard solutions of i) swertianolin, ii) demethylbellidifolin, and iii) bellidifolin were prepared to concentrations of $0.02 \mathrm{mg} / \mathrm{ml}$. HPLC analysis was performed on a XBridge C18 $(4.6 \times 250 \mathrm{~mm}, 5 \mu \mathrm{m}$, Waters Corporation) maintained at $35^{\circ} \mathrm{C}$. Mobile phases $\mathrm{A}$ and $\mathrm{B}$ were acetonitrile and $0.1 \%$ acetic acid, respectively. The flow rate was set at $1.0 \mathrm{ml} / \mathrm{min}$. The detection wavelength was set at $254 \mathrm{~nm}$ to identify xanthones from G. acuta (Fig. 1).

Statistical analysis. Each experiment was repeated three times. Data are expressed as the mean \pm standard error of the mean or mean \pm standard deviation. All statistical analyses were performed with SPSS 21.0 statistical software (IBM, Corp.). Differences were analyzed using one-way analysis of variance followed by Tukey's post hoc test. $\mathrm{P}<0.05$ was considered to indicate a statistically significant difference.

\section{Results}

G. acuta ameliorates ISO-induced myocardial fibrosis and cardiac remodeling in rats. Morphological examination of heart tissue sections stained with $\mathrm{H} \& \mathrm{E}$ revealed that an ISO-induced myocardial fibrosis rat model had been successfully established. Compared with the control group, heart tissues from ISO-treated rats exhibited widespread myocardial structural disorder and necrotic degeneration. Compared with the ISO group, G. acuta treatment produced a marked improvement in ISO-induced myocardial necrosis and structural disorder (Fig. 2A and B). Masson trichrome staining revealed that the collagen deposition was significantly increased in the
ISO-treated rat hearts. However, G. acuta treatment caused a significant reduction in collagen deposition compared with the ISO group ( $\mathrm{P}<0.05$; Fig. $2 \mathrm{~A}$ and $\mathrm{C})$. WGA staining indicated that ISO could significantly induce cardiomyocytes injury and hypertrophy $(\mathrm{P}<0.01)$, and treatment with $G$. acuta could inhibit myocardial hypertrophy (Fig. 2A and D). These results indicate that $G$. acuta treatment caused an inhibition of collagen deposition and ameliorated ISO-induced myocardial fibrosis and left ventricle remodeling.

G. acuta inhibits the expression of collagens I, III and $\alpha-S M A$ induced by ISO. In order to further confirm G. acuta's inhibitory effects on myocardial fibrosis, the content and distribution of collagen types I and III, the two major components of the ECM, were quantified using immunohistochemical staining and western blotting. Immunohistochemical staining revealed that the expression of myocardial collagens I and III were markedly enhanced in the ISO group vs. the control group. G. acuta treatment decreased ISO-induced collagens I and III expression enhancement (Fig. 3A-C). Western blotting results indicated that $G$. acuta treatment inhibited the collagens I and III expression (Fig. 3E and G). These findings demonstrate that $G$. acuta treatment caused a decrease in collagens I and III expression in the process of ISO-induced myocardial fibrosis.

A crucial event in myocardial fibrosis is the conversion of cardiac fibroblasts (CFs) to myofibroblasts (MFs). MFs express contractile proteins, including $\alpha$-SMA. Therefore, the expression of $\alpha$-SMA, which is a typical marker of MFs differentiation, was analyzed using immunohistochemical staining and western blotting. In the control group, $\alpha$-SMA-positive staining was observed in the blood vessels. In comparison with the control group (without ISO), an abundance of ISO-induced brown particles was present in the fibrotic areas, while G. acuta treatment ameliorated the ISO-induced positive brown particles (Fig. 3A and D). Western blotting results indicated that the expression level of $\alpha$-SMA was significantly downregulated by G. acuta ( $\mathrm{P}<0.01$; Fig. $3 \mathrm{~F}$ and $\mathrm{H})$.

These findings indicate that G. acuta treatment caused inhibition of ISO-induced myocardial fibrosis by reducing collagens I, III and $\alpha$-SMA expression in the heart tissue.

G. acuta treatment inhibits ISO-induced TGF- $\beta 1$ expression and the phosphorylation of T $\beta R I$ and II. TGF- $\beta 1$ is the major regulator of the fibrogenic process. In order to identify whether TGF- $\beta 1$ is involved in G. acuta-mediated attenuation of myocardial fibrosis, TGF- $\beta 1$ levels were examined in the heart tissue. Immunohistochemical analysis revealed that the expression levels of TGF- $\beta 1$ were increased in the ISO group compared with the control group. However, compared with the ISO group, G. acuta treatment caused a significant decrease in the ISO-induced TGF- $\beta 1$ expression levels $(\mathrm{P}<0.01$; Fig. 4A and B). Western blotting results showed that TGF- $\beta 1$ expression was significantly decreased in G. acuta treatment group $(\mathrm{P}<0.01$; Fig. 4C). These results indicate that $G$. acuta treatment inhibited ISO-induced TGF- $\beta 1$ expression in heart tissues.

Phosphorylation of the TGF- $\beta$ receptor is responsible for the initiation of multiple TGF- $\beta$ signaling pathways. In order to confirm whether $G$. acuta affects TGF- $\beta$ receptors, the 

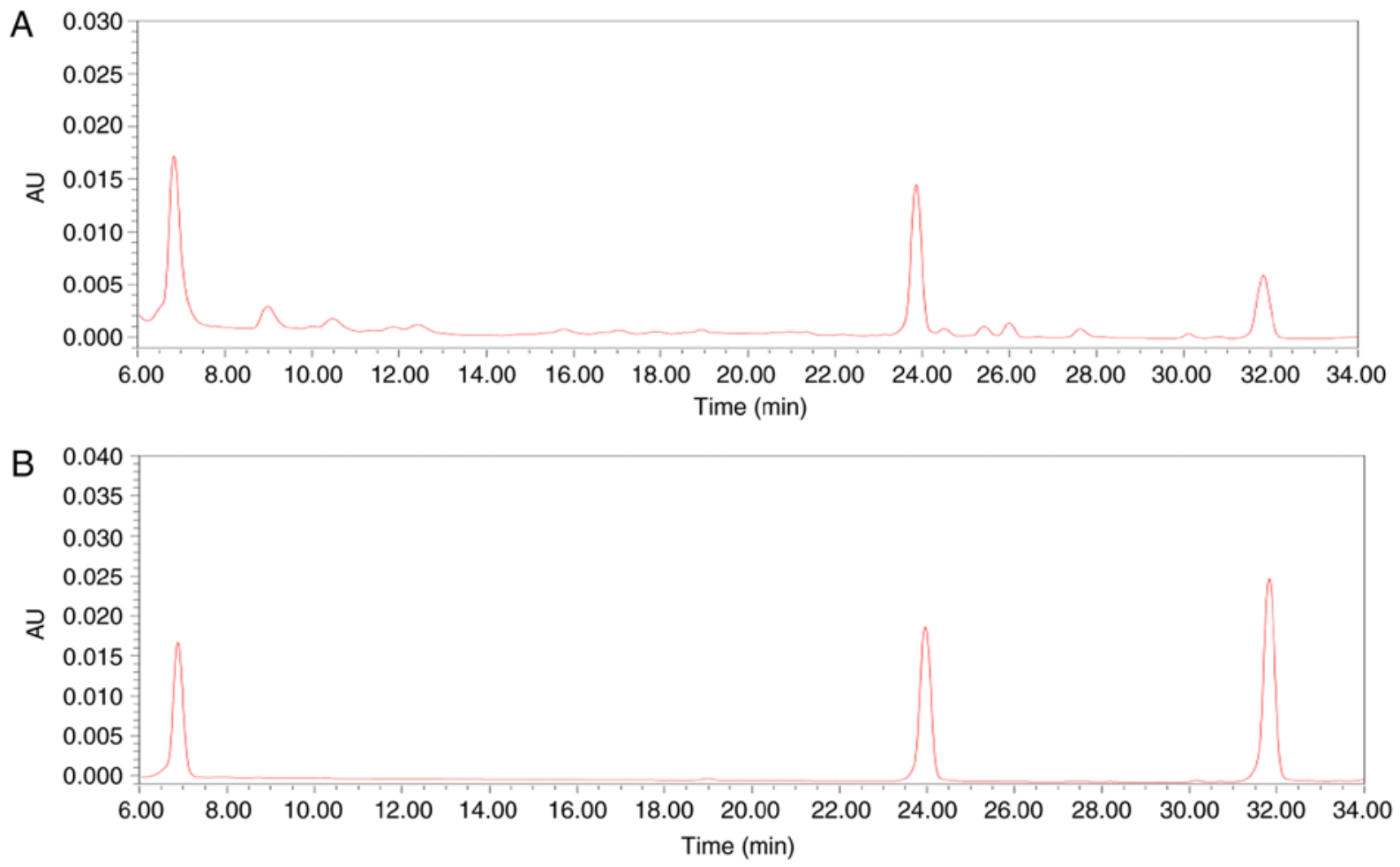

Figure 1. Analysis of xanthone compounds in G. acuta using high performance liquid chromatography. (A) The aqueous extracts of G. acuta. (B) The mixed standard solutions. The peaks were numbered as 1: Swertianolin, 2: Demethylbellidifolin and 3: Bellidifolin. G. acuta, Gentianella acuta.

A

H\&E
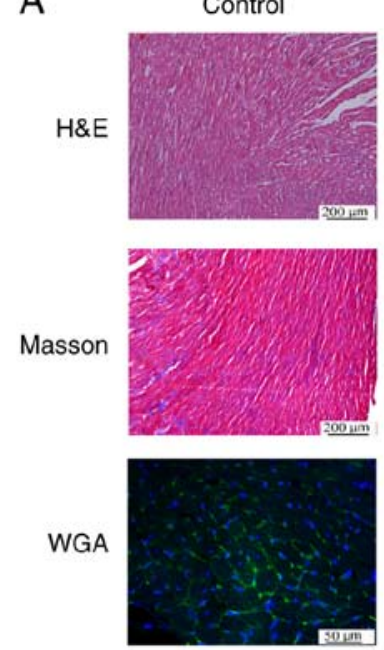

B

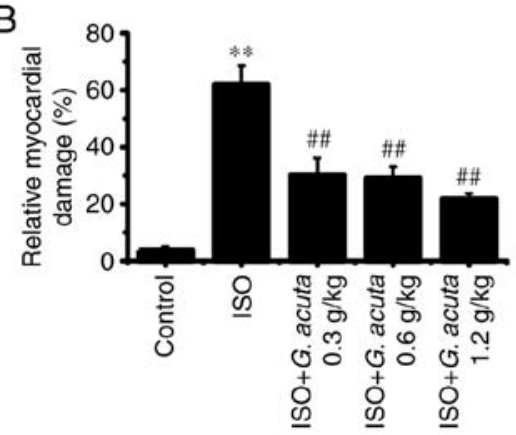

ISO
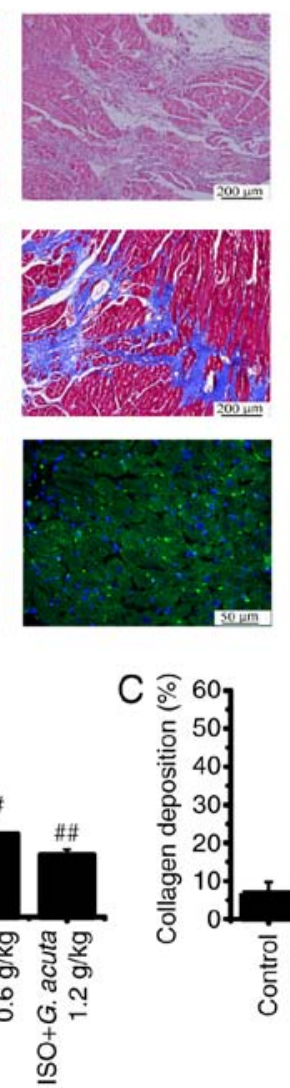

ISO+G. acuta $0.3 \mathrm{~g} / \mathrm{kg}$
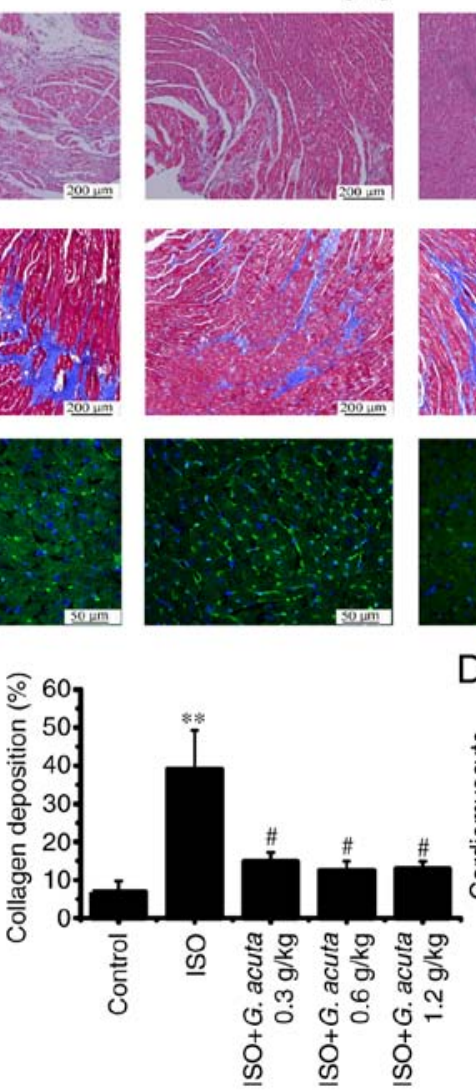
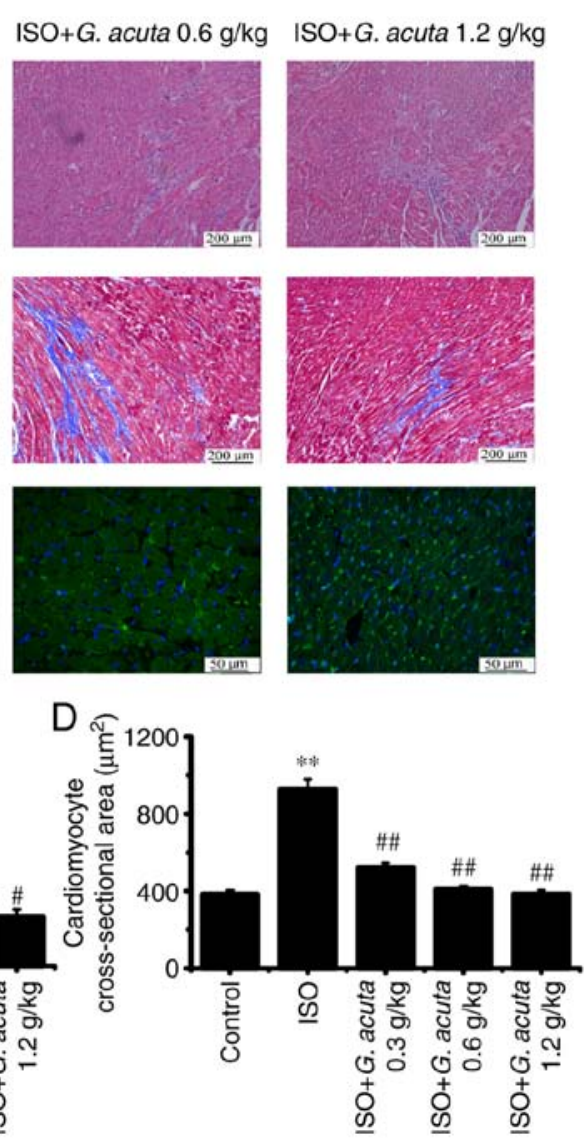

Figure 2. G. acuta ameliorates ISO-induced myocardial fibrosis and cardiac remodeling in rats. (A) H\&E staining to detect morphological changes; Masson trichrome staining to detect myocardial fibrosis; scale bars $=200 \mu \mathrm{m}$; WGA staining to access cardiomyocyte cross-sectional area; scale bars $=50 \mu \mathrm{m}$. (B) Bar graphs show the percentage of relative myocardial damage. (C) Bar graphs show the percentage of collagen deposition. (D) Bar graphs show the cardiomyocyte cross-sectional area. Data are shown as the mean \pm standard deviation. ${ }^{* *} \mathrm{P}<0.01$ vs. the Control, ${ }^{~} \mathrm{P}<0.05$ and ${ }^{\# \#} \mathrm{P}<0.01$ vs. ISO. ISO, isoproterenol; H\&E , hematoxylin and eosin; WGA, wheat germ agglutinin; G. acuta, Gentianella acuta. 
A

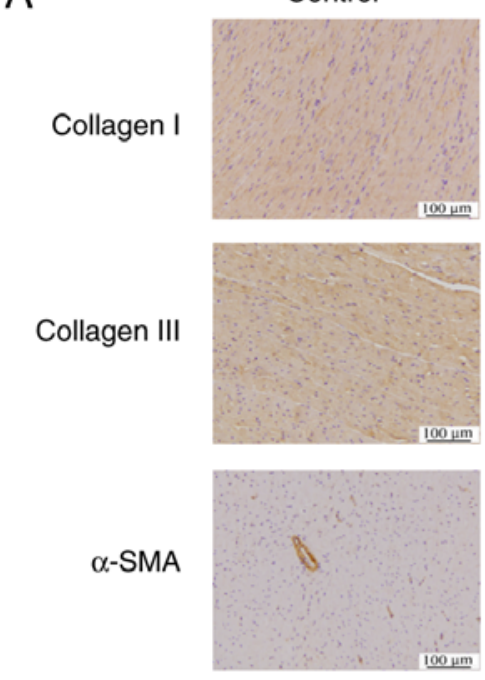

$\mathrm{B}$

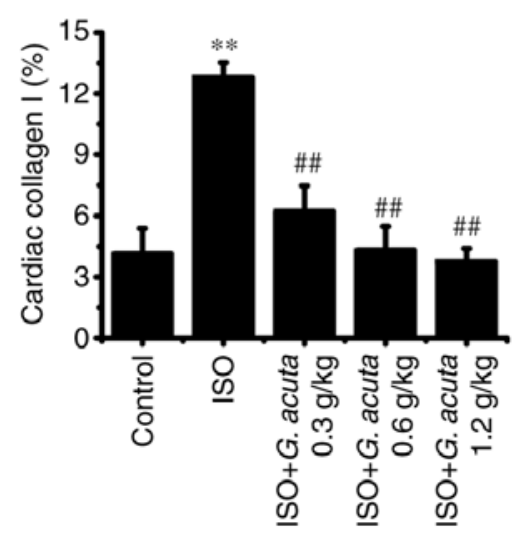

ISO
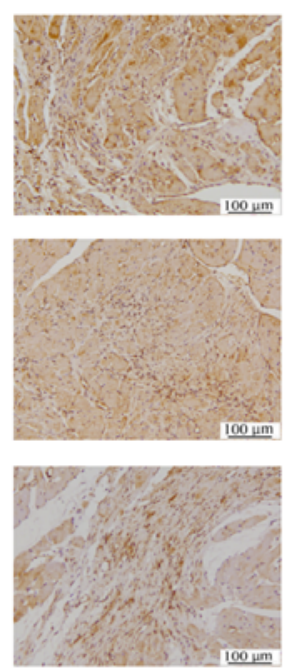

ISO+G. acuta $0.3 \mathrm{~g} / \mathrm{kg} \quad \mathrm{ISO}+$ G. acuta $0.6 \mathrm{~g} / \mathrm{kg}$ ISO+G. acuta $1.2 \mathrm{~g} / \mathrm{kg}$
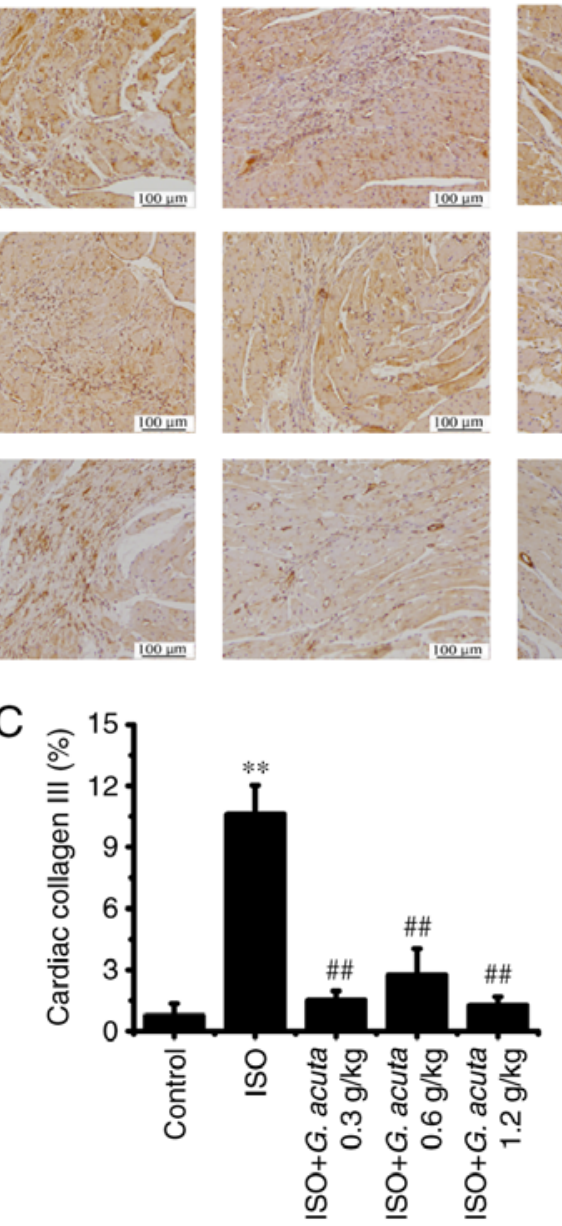
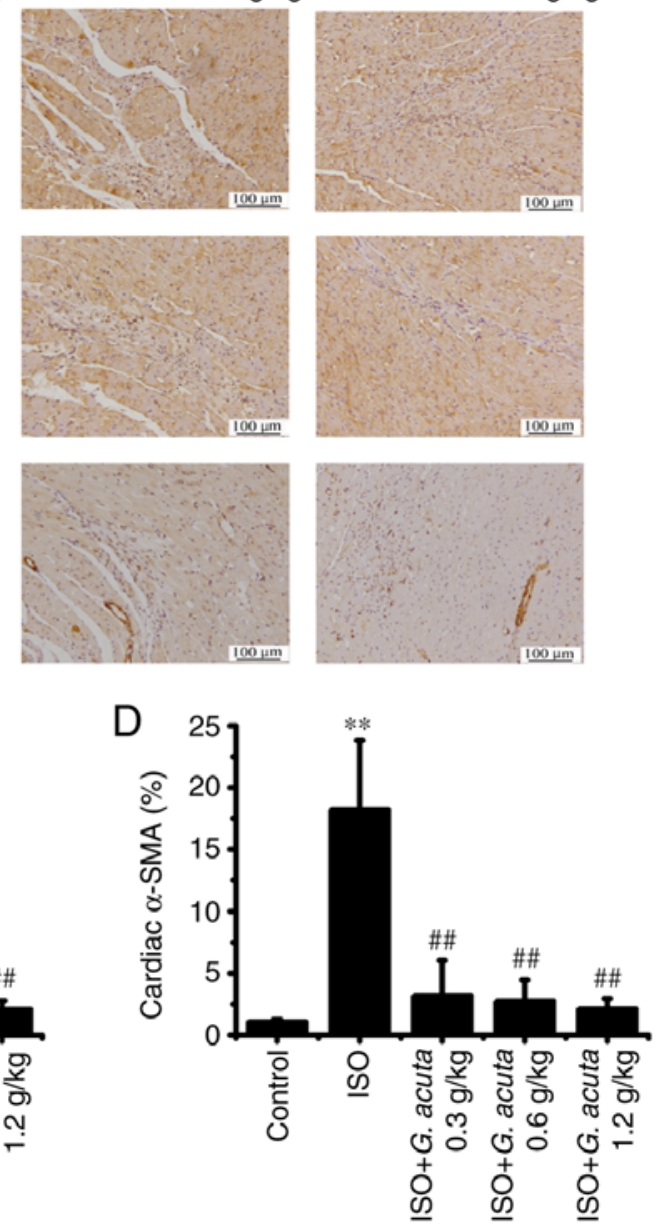
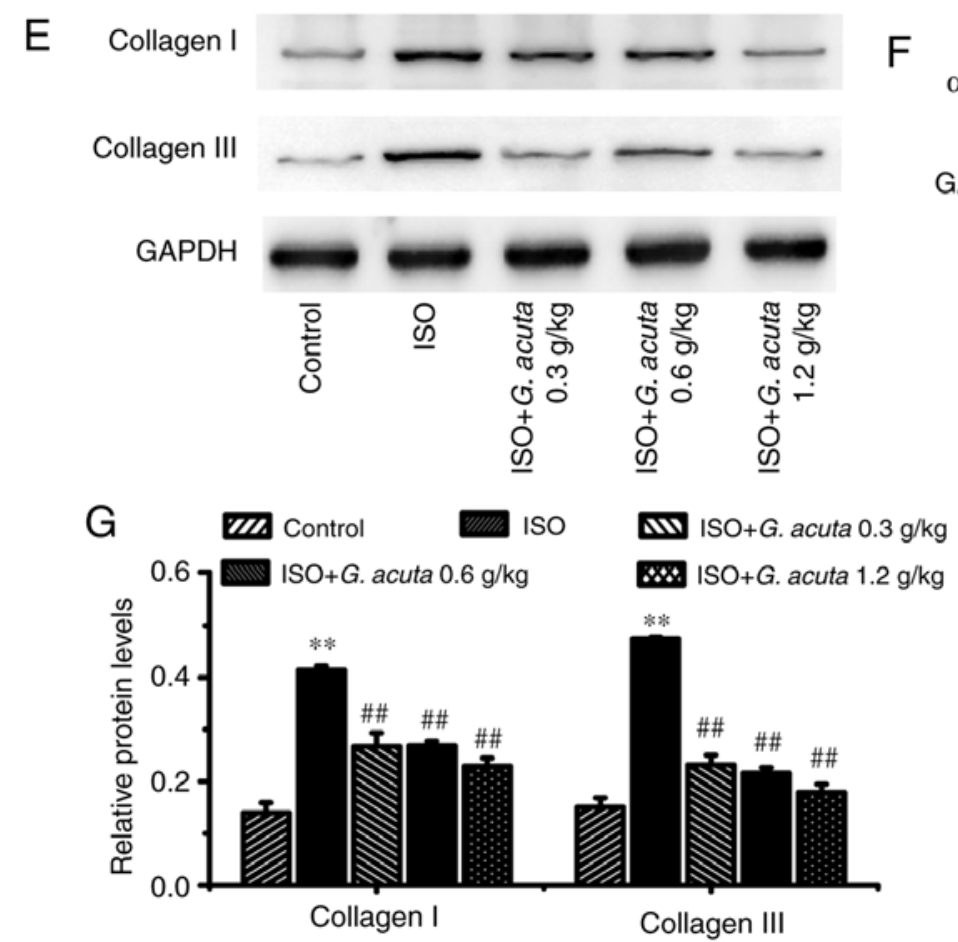

$\mathrm{F}$
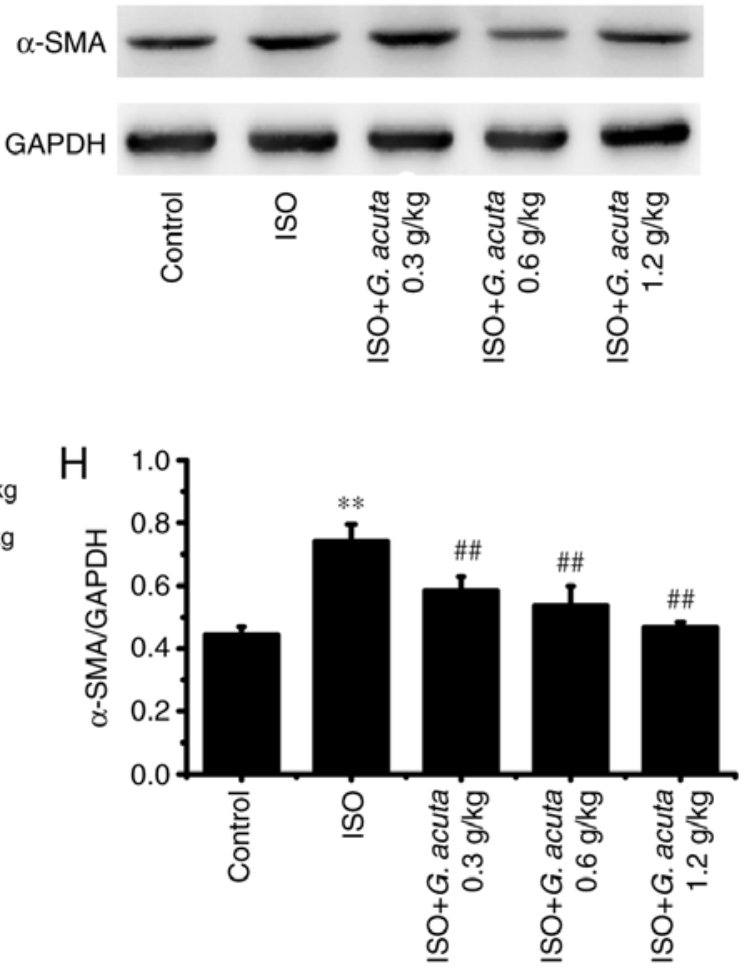

Figure 3. G. acuta inhibits the expression of collagens I, III and $\alpha$-SMA induced by ISO. (A) Immunohistochemical staining for collagens I, III and $\alpha$-SMA, scale bars $=100 \mu \mathrm{m}$. Bar graphs show the percentage of (B) collagens I, (C) III and (D) $\alpha$-SMA. (E) Western blotting detection of collagens I, III and (F) $\alpha$-SMA. (G) Bar graphs show fold-changes for the collagens I and III and (H) $\alpha$-SMA expression as analyzed by western blotting. GAPDH was used as a loading control $(\mathrm{n}=3)$. Data are shown as the mean \pm standard error of the mean. ${ }^{* *} \mathrm{P}<0.01$ vs. the Control, ${ }^{\# \#} \mathrm{P}<0.01$ vs. ISO. $\alpha$-SMA, $\alpha$-smooth muscle actin; ISO, isoproterenol; G. acuta, Gentianella acuta . 
A

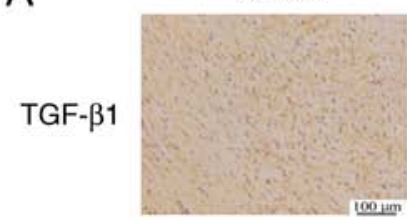

B
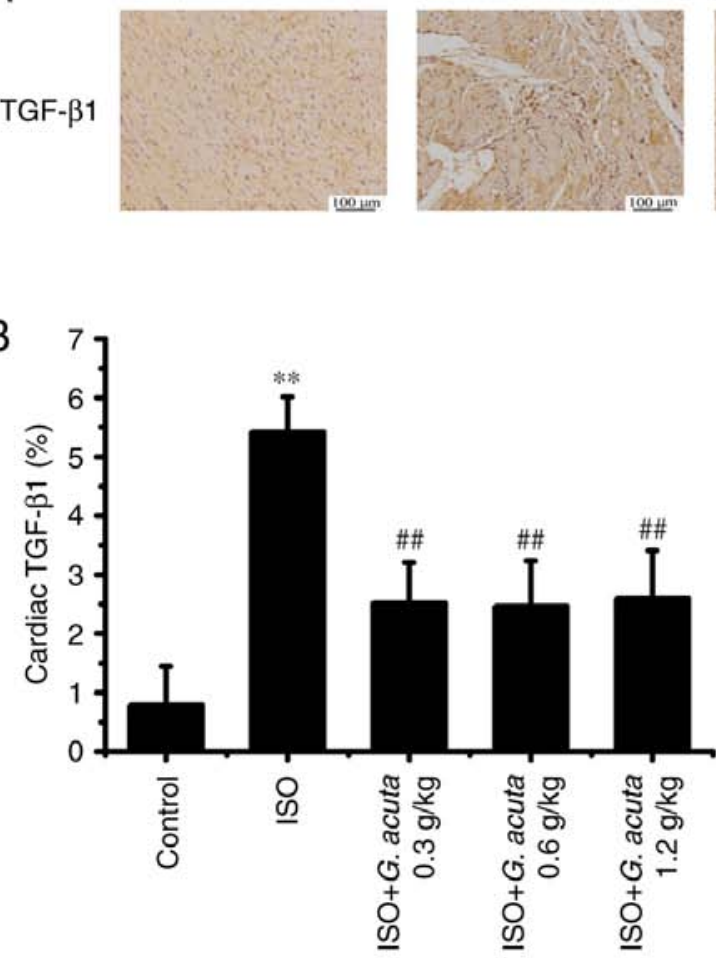

ISO+G. acuta $0.3 \mathrm{~g} / \mathrm{kg} I S O+G$. acuta $0.6 \mathrm{~g} / \mathrm{kg} I S O+G$. acuta $1.2 \mathrm{~g} / \mathrm{kg}$
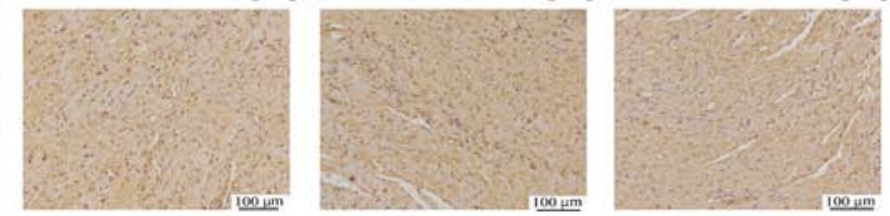

C
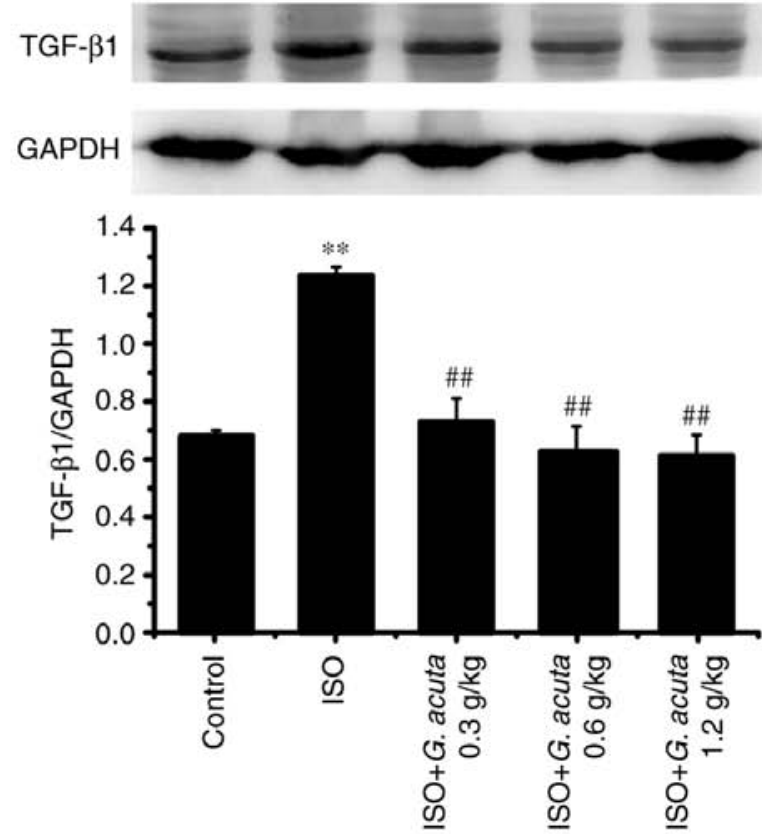

Figure 4. G. acuta causes a reduction in ISO-induced TGF- $\beta 1$ expression. (A) Immunohistochemical staining to detect TGF- $\beta 1$, scale bars=100 $\mu \mathrm{m}$. (B) Bar graphs show the percentage of TGF- $\beta 1$. (C) Western blotting to detect TGF- $\beta 1$ and bar graphs show fold-changes of TGF- $\beta 1$ expression as analyzed by western blotting. GAPDH was used as a loading control $(\mathrm{n}=3)$. Data are shown as the mean \pm standard error of the mean. ${ }^{* *} \mathrm{P}<0.01$ vs. the Control, ${ }^{\# \#} \mathrm{P}<0.01$ vs. ISO. TGF- $\beta 1$, transforming growth factor; ISO, isoproterenol; G. acuta, Gentianella acuta.

expression and phosphorylated levels of T $\beta$ RI and II were examined by western blotting. The results indicated that T $\beta R I$ expression and levels of T $\beta$ RI and II phosphorylation were significantly upregulated in the ISO group compared with the control group $(\mathrm{P}<0.01)$. Moreover, G. acuta treatment abolished upregulation of these ISO-induced proteins expression and phosphorylation levels (Fig. 5). These results demonstrate that $G$. acuta attenuated T $\beta \mathrm{RI}$ expression and inhibited the phosphorylation and activation of T $\beta$ RI and II.

G. acuta inhibits ISO-activated TGF- $\beta 1 /$ smads signaling. The TGF- $\beta 1 /$ Smads signaling pathway plays a critical role in myocardial fibrosis. Smad proteins are the primary downstream molecules of T $\beta$ RI. In order to investigate whether G. acuta caused a reduction in the ISO-induced expression and activation of Smads, immunohistochemical staining and western blotting were performed. In comparison with the control group, the immunoreactivities of Smads2, 3 and 4 were significantly increased in the ISO group. Moreover, $G$. acuta treatment reduced the increase in ISO-induced Smads2, 3 and 4 expression (Fig. 6A). Western blot analysis showed that G. acuta treatment caused a decrease in the Smads2 and 4 expression. In addition, compared with the control group, Smads 2 and 3 phosphorylation levels were significantly increased in the ISO group $(\mathrm{P}<0.01)$. G. acuta treatment caused a significant reduction in Smads 2 and 3 phosphorylation levels, compared with the ISO group
(P<0.05; Fig. 6B-E). These results demonstrate that G. acuta treatment attenuated Smads 2 and 4 expression and inhibited activation of ISO-induced Smads 2 and 3 via phosphorylation. In conclusion, the present data suggested that $G$. acuta caused an inhibition of myocardial fibrosis through the regulation of the TGF- $\beta 1 /$ Smads signaling pathway (Fig. 7).

\section{Discussion}

In mammals, the ability to regenerate the heart after birth is limited and the adult mammalian heart rarely exhibits the capacity for self-renewal. Myocardial fibrosis is a morphological feature of ventricular remodeling in a variety of heart diseases involved in myocardial infarction (23). Accompanying the fibrosis, normal structural components of heart tissue can be disrupted. Employment of ISO has been popular for the induction of myocardial fibrosis in rats. A previous study showed that the pathophysiological change of left ventricular induced by ISO could persist up to 21 days after a single subcutaneous injection (24). In the authors' previous study, it was confirmed that $G$. acuta could decrease ISO-increased heart rate, improve cardiac function and ameliorate myocardial fibrosis (18). However, the detailed mechanism of G. acuta on myocardial fibrosis was not clear. In this study, H\&E and Masson trichrome staining demonstrated that G. acuta alleviated cardiac structural disorder and myocardial fibrosis induced by ISO, which were consistent 
A

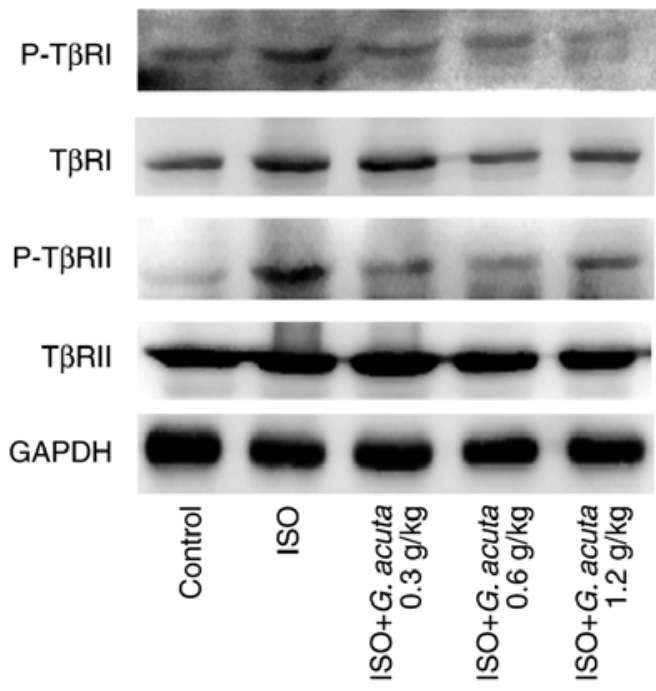

C

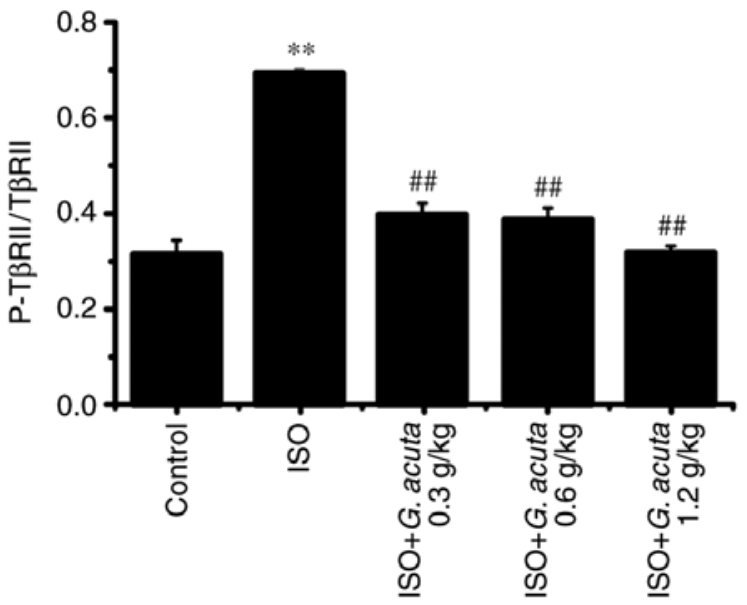

B

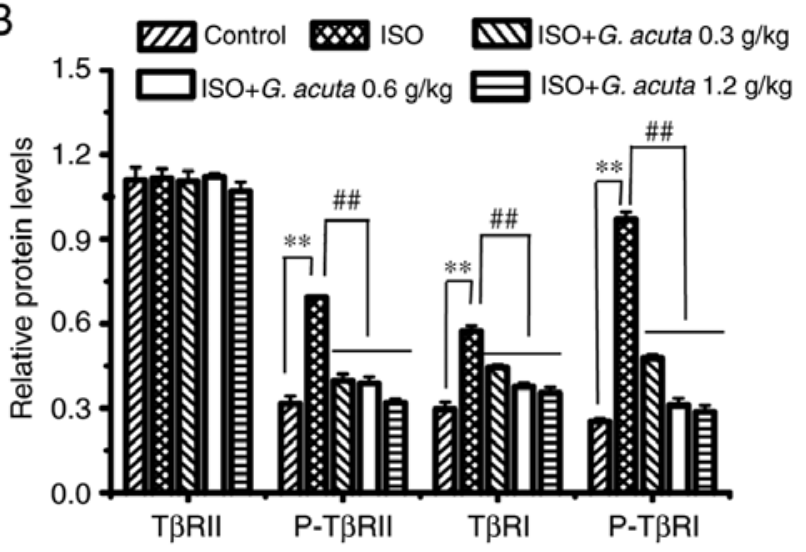

D

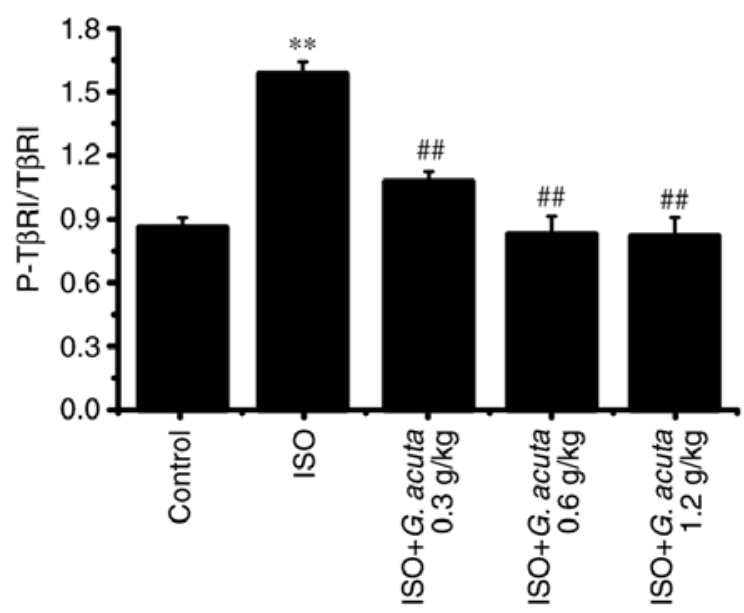

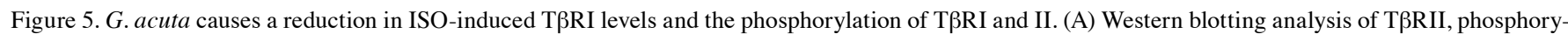
lated (P)-T $\beta R I I, T \beta R I$, P-T $\beta R I$. (B) Bar graphs show fold-changes of the T $\beta$ RII, P-T $\beta R I I, T \beta R I$, P-T $\beta$ RI expression, as analyzed by western blotting. GAPDH was used as a loading control ( $n=3$ ). (C) Bar graphs show fold-changes of P-T $\beta R I I / T \beta R I I$ and (D) P-T $\beta R I / T \beta R I$. Data are shown as mean \pm standard error of the mean. ${ }^{* *} \mathrm{P}<0.01$ vs. the Control and ${ }^{\# \#} \mathrm{P}<0.01$ vs. ISO. ISO, isoproterenol; G. acuta, Gentianella acuta; $\mathrm{P}-\mathrm{T} \beta \mathrm{R}$, phosphorylated- TGF- $\beta$ receptor.

with the authors' previous study (18). In addition, WGA staining determined that G. acuta also inhibited ISO-induced myocardial hypertrophy. These results determined that G. acuta treatment ameliorated ISO-induced myocardial fibrosis and ventricular remodeling.

ECM is a crucial element and regulator in numerous biological tissues (25). In the heart, the collagen matrix plays an important role in supporting muscle cells and blood vessels via connecting cells and muscle to govern the architecture and maintain an important diastolic and systolic myocardial stiffness $(26,27)$. Adverse cardiac remodeling and myocardial fibrosis lead to the alteration of ECM in heart tissue. Fibrillar collagen replaces dead cardiomyocytes and scar tissue formation occurs in the infarct zone along with the excessive deposition of collagen in the interstitium remote to the infarction site (28). One of the key events in myocardial fibrosis is the transformation of myocardial CFs into MFs. MFs are the primary cells involved in the repair of pathological tissue, which produce collagen during the fibrotic process $(29,30)$. High $\alpha$-SMA expression is a key MFs feature (31). In this study, it was determined that ISO induced the excessive deposition of collagen and G. acuta decreased the collagen deposition by immunohistochemical staining, that results were in consistent with the authors' previous study (18). It was also verified that $G$. acuta downregulated collagen expression by western blotting. Furthermore, G. acuta treatment ameliorated the expression of $\alpha$-SMA in the context of ISO-induced myocardial fibrosis. These data suggest that G. acuta inhibited the conversion of CFs to MFs, reduced collagen synthesis and improved myocardial fibrosis.

TGF- $\beta$ family members consist of three isoforms (TGF- $\beta 1$, TGF- $\beta 2$ and TGF- $\beta 3$ ), which are involved in numerous cellular processes, including cell differentiation, organ development, wound healing and immune regulation (10). However, the distinct biological functions of different isoforms in vivo are still under debate. Accumulating evidence has revealed that TGF- $\beta 1$ plays a vital role in fibrogenesis and myocardial remodeling (32). TGF- $\beta 1$ mediates the process of ECM production, which can induce $\mathrm{CF}$ differentiation and promote collagen synthesis (33). In this study, the present results showed that TGF- $\beta 1$ expression was upregulated by ISO in the heart, which was attenuated by G. acuta treatment. The results were 
A
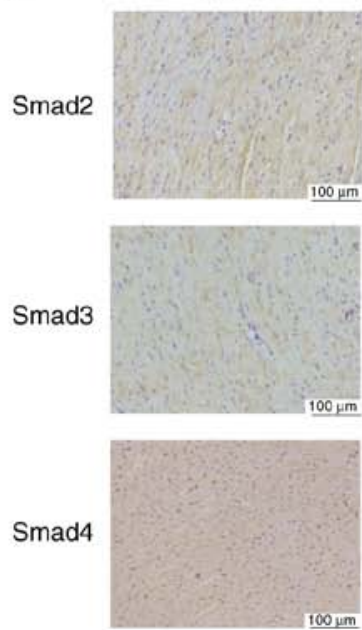

B

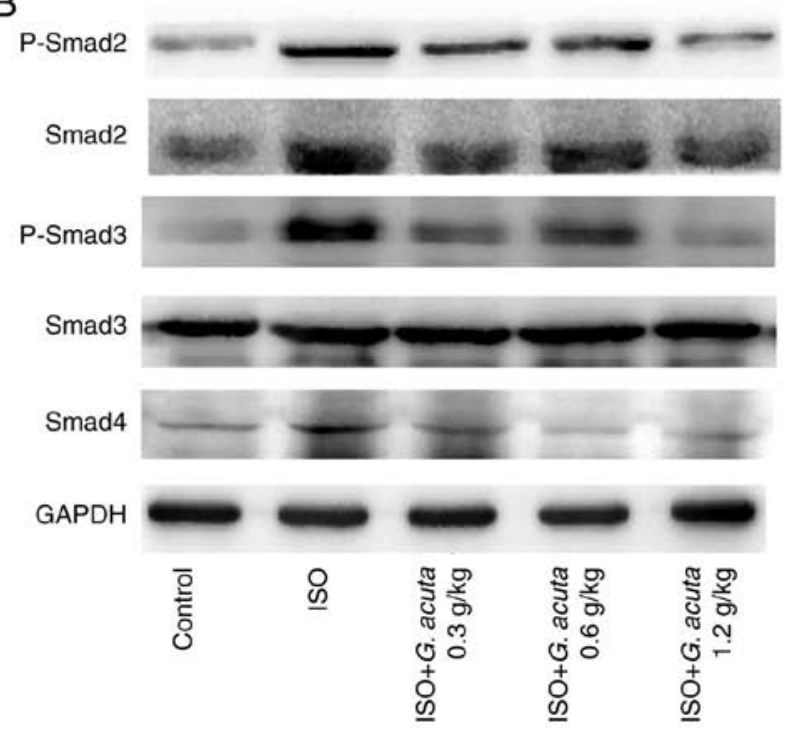

ISO+G. acuta $0.3 \mathrm{~g} / \mathrm{kg} \mathrm{ISO}+\mathrm{G}$. acuta $0.6 \mathrm{~g} / \mathrm{kg}$
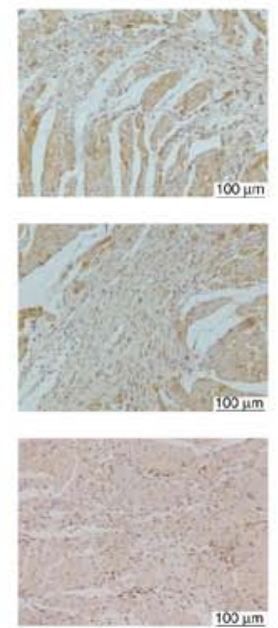
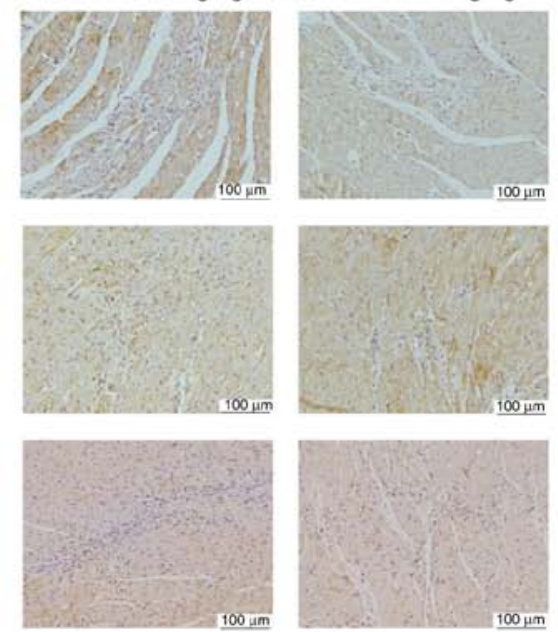

ISO+G. acuta $1.2 \mathrm{~g} / \mathrm{kg}$
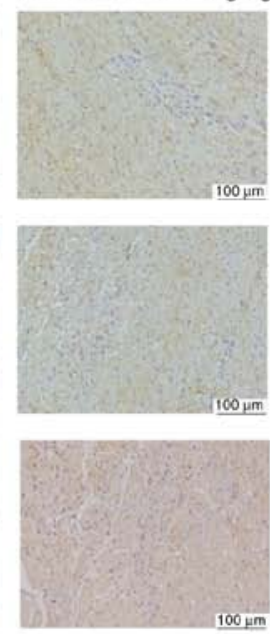
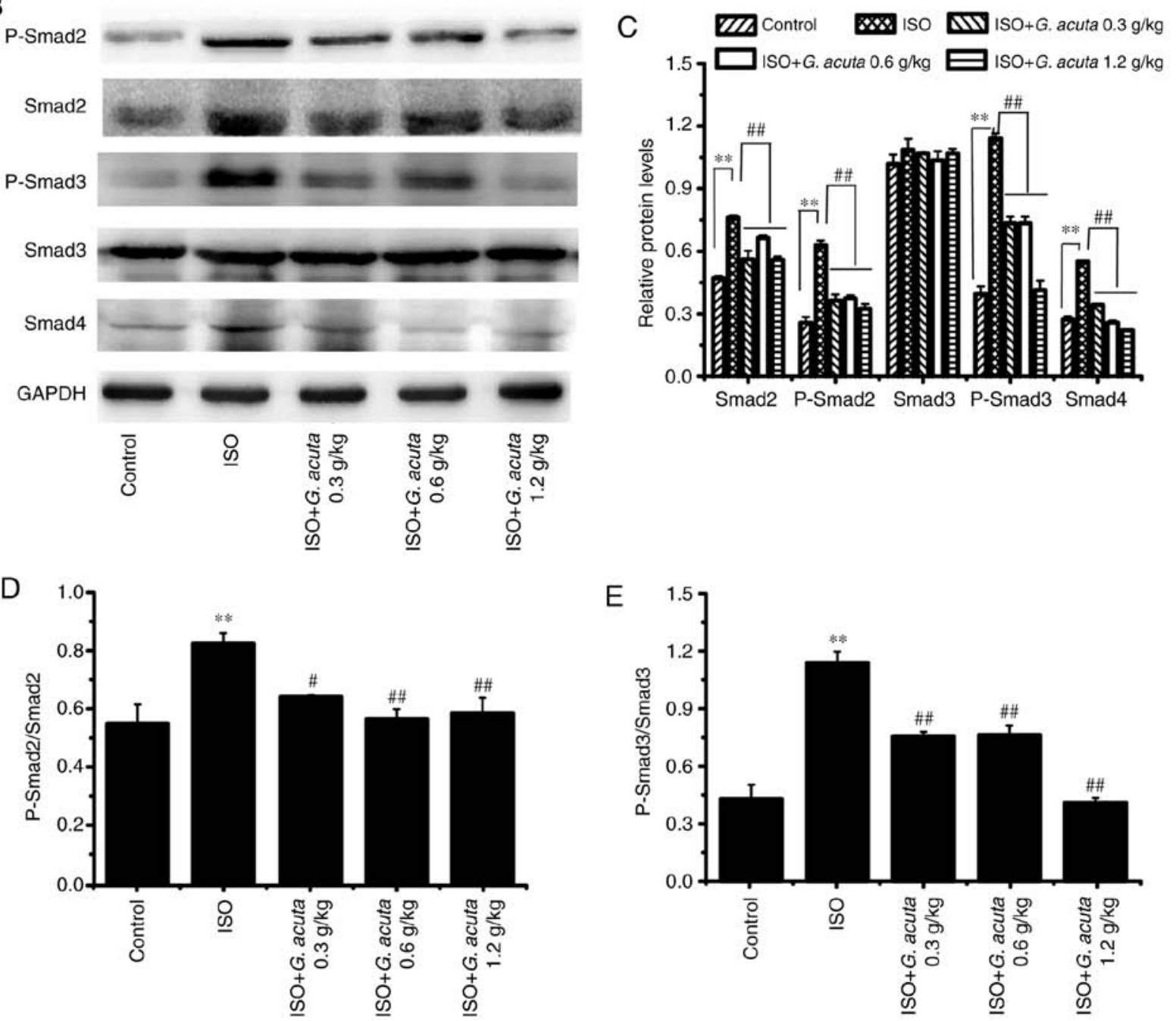

Figure 6. G. acuta causes inhibition of ISO-activated TGF- $\beta 1 /$ Smads signaling. (A) Immunohistochemistry staining to detect Smads2, 3 and 4 ; scale bars $=100 \mu \mathrm{m}$. (B) Western blotting analysis of Smad2, P-Smad2, Smad3, P-Smad3 and Smad4 expression. (C) Bar graphs show fold-changes of Smad2, P-Smad2, Smad3, P-Smad3 and Smad4 expression as analyzed by western blotting. GAPDH was used as a loading control (n=3). (D) Bar graphs show fold-changes of P-Smad2/Smad2 and (E) P-Smad3/Smad3. Data are shown as mean \pm standard error of the mean. ${ }^{* *} \mathrm{P}<0.01 \mathrm{vs}$. control, ${ }^{\#} \mathrm{P}<0.05$ and ${ }^{\# \#} \mathrm{P}<0.01$ vs. ISO. P-Smad, phosphorylated-mothers against decapentaplegic homolog; ISO, isoproterenol; G. acuta, Gentianella acuta.

consistent with the authors' previous study (18). Therefore, anti-fibrotic effects of G. acuta may be mediated by inhibiting the TGF- $\beta 1$ signaling at least partly.
TGF- $\beta 1$ signaling regulates tissue fibrosis and tissue scarring by activating specific downstream serine/threonine kinase receptors. T $\beta R I I$ is the specific downstream serine/threonine 


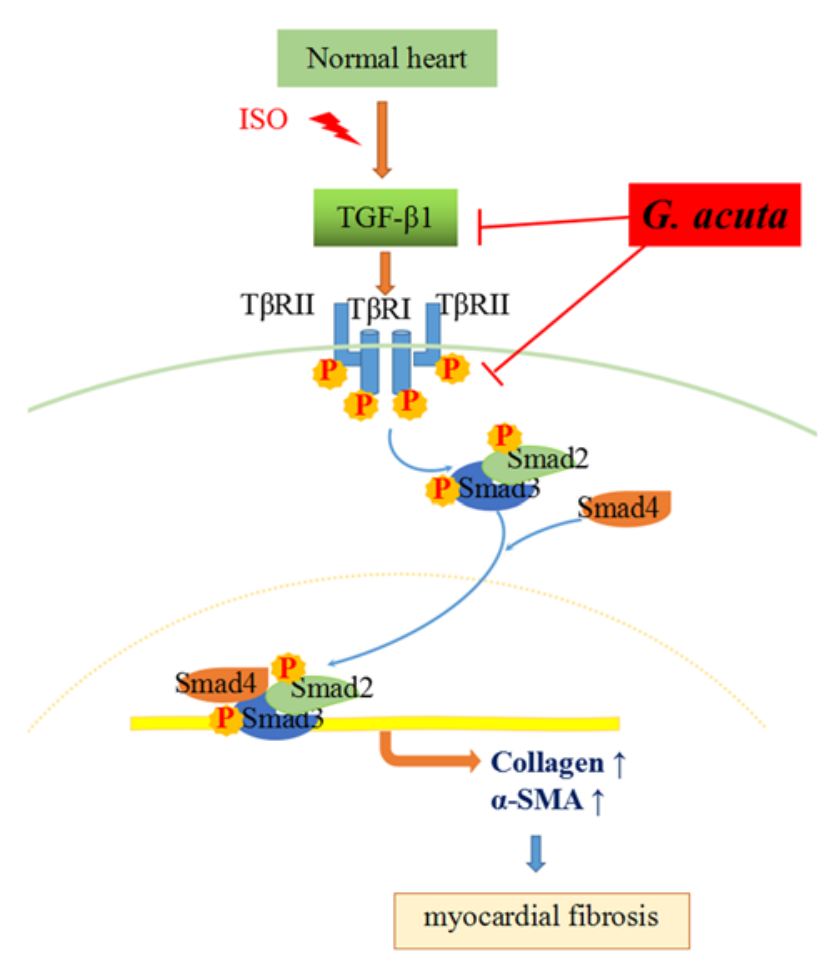

Figure 7. The proposed antifibrotic mechanism of G. acuta in ISO-induced myocardial fibrosis in rats. Myocardial injury activates the TGF- $\beta /$ Smads pro-fibrotic signaling pathway. G. acuta inhibits TGF- $\beta 1$ expression, downregulates T $\beta$ RI expression and T $\beta$ RI and II phosphorylation levels, suppresses Smad expression and phosphorylation activation, and causes a decrease in ISO-induced $\alpha$-SMA, collagens I and III expression in heart tissues. The results indicate that $G$. acuta improves ISO-induced myocardia fibrosis via inhibition of the TGF- $\beta 1 /$ Smads signaling pathway. P-Smad, phosphorylated-mothers against decapentaplegic homolog; ISO, isoproterenol; G. acuta, Gentianella acuta; P-T $\beta$ R, phosphorylated- TGF- $\beta$ receptor.

kinase receptor of TGF- $\beta 1$. TGF- $\beta 1$ combines with T $\beta$ RII, which results in T $\beta$ RII autophosphorylation (34). It is essential for its kinase activity and regulation of downstream signaling to regulate tissue fibrosis and tissue scarring. As a result, the activated T $\beta$ RII further binds and phosphorylates T $\beta$ RI (32). Activation of this receptor complex leads to downstream signaling molecule activation and then signal transmission into the cells. In the present study, G. acuta treatment alleviated the ISO-induced T $\beta$ RI and II phosphorylation. These results indicated that $G$. acuta could block the TGF- $\beta$ signaling pathway activation.

TGF- $\beta$ signaling includes canonical and non-canonical (Smad and non-Smad-based, respectively) signaling pathways (12). Numerous studies have demonstrated that the Smad-dependent pathway contributes to myocardial fibrosis in the TGF- $\beta$-induced signaling pathway $(10,12,35,36)$. Smad proteins are the core downstream signaling molecules of TGF- $\beta 1$ and they are mediated by the TGF- $\beta$ receptor. Smads 2 and 3 can be phosphorylated and activated. The latter becomes a transcription complex by further combining with Smad4. After this event, the complex moves into the nucleus to complete the process of intracellular signaling transduction via mediation of the target genes such as collagen and $\alpha$-SMA, leading to myocardial fibrosis (37). In the present study, it was demonstrated that G. acuta caused a decrease in Smads 2 and 3 phosphorylation levels and inhibited Smads 2 and 4 expression in ISO-induced myocardial fibrosis. It was confirmed that G. acuta inhibited myocardial fibrosis via TGF- $\beta 1 /$ Smads signaling pathway suppression.

An increasing number of studies have demonstrated that $G$. acuta exerts a protective effect on the heart $(15,16)$. In the authors' previous study, it was found that $G$. acuta caused inhibition of myocardial fibrosis (18). In the present study, the mechanism underlying the anti-fibrotic effect was further clarified to be based upon the TGF- $\beta 1 / \mathrm{Smads}$ signaling pathway. Phytochemical analysis showed that G. acuta contains numerous kinds of constituents, such as xanthones, flavonoids, monoterpenes, lignans and phenolic acids, and xanthones were elucidated to be main constituents in the plant (14-16,38,39). Recently, a few studies have explored the effects of the primary active components of this plant on anti-myocardial injury (14-16) and xanthones from G. acuta can protect cardiomyocytes from injury $(16,40)$. The present study first revealed that the aqueous extracts of G. acuta, a form of clinical application, contains three xanthones components by HPLC (13). The above results indicated that xanthones were a kind of major bioactivity substances in G. acuta. However, except for xanthones, it has been reported that phenolics, flavonoids and lignan glycosides also have a protective effect on the heart $(17,41)$. Therefore, the effect of G. acuta on myocardial fibrosis may be the potential synergistic effect of various compounds. In addition, because of that, it is difficult to find a suitable plant drug or single compound as a positive control that was the main limitation of the present study. Based upon the present study, it was hypothesized that the inhibitor of $T \beta R$ may be a potential positive control, such as Galunisertib or LY2109761, which exhibited preclinical anti-fibrotic effects $(42,43)$.

For the first time, the present study demonstrated that G. acuta treatment causes a reduction in TGF- $\beta 1$ and T $\beta$ RI expression and T $\beta$ RI and II phosphorylation levels, thereby suppressing the Smads2 and 4 expression and Smads2 and 3 activation. This suppression further inhibits the ISO-induced $\alpha$-SMA and collagens I and III expression in rats. These findings demonstrate that $G$. acuta has potential anti-fibrotic function via inhibition of the TGF- $\beta 1 /$ Smads signaling transduction pathway. It will be necessary to confirm the specific active ingredients that are responsible for the therapeutic effects of G. acuta.

\section{Acknowledgements}

Not applicable.

\section{Funding}

The present study was supported by grants from the National Natural Science Foundation of China (grant nos. 81573698 and 31271466), the Science Foundation for Youth of the Higher Education Institutions of Hebei Province (grant no. 2016137), the Projects of Hebei Provincial Administration of Traditional Chinese Medicine (grant nos. 2016003 and 2012080) and the Graduate Innovation Funding Projects of Hebei Province (grant no. CXZZBS2018155). 


\section{Availability of data and materials}

The datasets used and/or analyzed during the current study are available from the corresponding authors on reasonable request.

\section{Authors' contributions}

As the corresponding author, AL contributed to the conception and design of the study. HY contributed to the study's design, performed the experiments, analyzed the data and prepared the manuscript for writing. GX performed western blot analysis. JHS established the experimental animal model. CZ contributed significantly to HPLC analysis and identification of the effective constituent xanthones. YZ, JNS, YFL and YL were involved in analyzing the data.

\section{Ethics approval and consent to participate}

All experiments were approved by the Institutional Animal Care and Use Committee of Hebei University of Chinese Medicine (Shijiazhuang, China).

\section{Patient consent for publication}

Not applicable.

\section{Competing interests}

The authors declare no competing interests.

\section{References}

1. Masci PG, Schuurman R, Andrea B, Ripoli A, Coceani M, Chiappino S, Todiere G, Srebot V, Passino C, Aquaro GD, et al: Myocardial fibrosis as a key determinant of left ventricular remodeling in idiopathic dilated cardiomyopathy: A contrast-enhanced cardiovascular magnetic study. Circ Cardiovasc Imaging 6: 790-799, 2013.

2. Dixon JA and Spinale FG: Pathophysiology of myocardial injury and remodeling: Implications for molecular imaging. J Nucl Med 51 (Suppl3r 1): 102S-106S, 2010.

3. Li L, Zhao Q and Kong W: Extracellular matrix remodeling and cardiac fibrosis. Matrix Biol 68-69: 490-506, 2018.

4. Frangogiannis NG: Cardiac fibrosis: Cell biological mechanisms, molecular pathways and therapeutic opportunities. Mol Aspects Med 65: 70-99, 2019.

5. Czubryt MP: Common threads in cardiac fibrosis, infarct scar formation, and wound healing. Fibrogenesis Tissue Repair 5: 19, 2012.

6. Meyers TA and Townsend D: Early right ventricular fibrosis and reduction in biventricular cardiac reserve in the dystrophin-deficient mdx heart. Am J Physiol Heart Circ Physiol 308: H303-H315, 2015.

7. Raman B, Ariga R, Spartera M, Sivalokanathan S, Chan K, Dass S, Petersen SE, Daniels MJ, Francis J, Smillie R, et al: Progression of myocardial fibrosis in hypertrophic cardiomyopathy: Mechanisms and clinical implications. Eur Heart J Cardiovasc Imaging 20: 157-167, 2019.

8. Goumans MJ and Ten Dijke P: TGF- $\beta$ signaling in control of cardiovascular function. Cold Spring Harb Perspect Biol 10: a022210, 2018

9. Yue Y, Meng K, Pu Y and Zhang X: Transforming growth factor beta (TGF- $\beta$ ) mediates cardiac fibrosis and induces diabetic cardiomyopathy. Diabetes Res Clin Pract 133: 124-130, 2017.

10. Hu HH, Chen DQ, Wang YN, Feng YL, Cao G, Vaziri ND and Zhao YY: New insights into TGF- $\beta /$ Smad signaling in tissue fibrosis. Chem Biol Interact 292: 76-83, 2018.
11. Leask A: Potential therapeutic targets for cardiac fibrosis: TGFbeta, angiotensin, endothelin, CCN2, and PDGF, partners in fibroblast activation. Circ Res 106: 1675-1680, 2010.

12. Gyorfi AH, Matei AE and Distler JHW: Targeting TGF- $\beta$ signaling for the treatment of fibrosis. Matrix Biol 68-69: 8-27, 2018.

13. Wunir, Chunliang and Khasbagan: Ewenki folk medicinal plants and its comparison with Mongolian medicine. Chin J Ethnomed Ethnopharm 18: 156-158, 2009 (In Chinese).

14. Liu Y, Ni Y, Ruan J, Qu L, Yu H, Han L, Zhang Y and Wang T: Bioactive gentixanthone and gentichromone from the whole plants of Gentianella acuta (Michx.) Hulten. Fitoterapia 113: 164-169, 2016.

15. Wang Z, Wu G, Liu H, Xing N, Sun Y, Zhai Y, Yang B, Kong AT, Kuang $\mathrm{H}$ and Wang Q: Cardioprotective effect of the xanthones from Gentianella acuta against myocardial ischemia/reperfusion injury in isolated rat heart. Biomed Pharmacother 93: 626-635, 2017.

16. Wang Z, Wu G, Yu Y, Liu H, Yang B, Kuang H and Wang Q: Xanthones isolated from Gentianella acuta and their protective effects against $\mathrm{H}_{2} \mathrm{O}_{2}$-induced myocardial cell injury. Nat Prod Res 32: 2171-2177, 2018.

17. Yu Y, Wang ZB, Zhai YD, Song PY, Wang QH, Yang BY and Kuang H: Lignan glycosides from Gentianella acuta (Michx.) Hulten and their protective effects against $\mathrm{H}_{2} \mathrm{O}_{2}$-induced apoptosis in H9c2 cardiomyoblast. Rec Nat Prod 8: 234-241, 2014.

18. Li AY, Wang JJ, Yang SC, Zhao YS, Li JR, Liu Y, Sun JH, An LP, Guan P and Ji ES: Protective role of Gentianella acuta on isoprenaline induced myocardial fibrosis in rats via inhibition of NF- $\kappa$ B pathway. Biomed Pharmacother 110: 733-741, 2019.

19. Xu SY, Bian RL and Chen X: Experimental methodology of pharmacology. People's Medical Publishing House, Beijing, 1982.

20. Blanchard OL and Smoliga JM: Translating dosages from animal models to human clinical trials-revisiting body surface area scaling. FASEB J 29: 1629-1634, 2015.

21. Zhou H, Chen X, Chen L, Zhou X, Zheng G, Zhang H, Huang W and Cai J: Anti-fibrosis effect of scutellarin via inhibition of endothelial-mesenchymal transition on isoprenaline-induced myocardial fibrosis in rats. Molecules 19: 15611-15623, 2014.

22. Wan Y, Xu L, Wang Y, Tuerdi N, Ye M and Qi R: Preventive effects of astragaloside IV and its active sapogenin cycloastragenol on cardiac fibrosis of mice by inhibiting the NLRP3 inflammasome. Eur J Pharmacol 833: 545-554, 2018.

23. Chen J, Zhan Y, Wang Y Han D, Tao B, Luo Z, Ma S, Wang Q, Li X, Fan L, et al: Chitosan/silk fibroin modified nanofibrous patches with mesenchymal stem cells prevent heart remodeling post-myocardial infarction in rats. Acta Biomater 80: 154-168, 2018.

24. Koga M, Kuramochi M, Karim MR, Izawa T, Kuwamura M and Yamate J: Immunohistochemical characterization of myofibroblasts appearing in isoproterenol-induced rat myocardial fibrosis. J Vet Med Sci 81: 127-133, 2019.

25. Daley WP and Yamada KM: ECM-modulated cellular dynamics as a driving force for tissue morphogenesis. Curr Opin Genet Dev 23: 408-414, 2013.

26. Gyöngyösi M, Winkler J, Ramos I, Do QT, Firat H, McDonald K, González A, Thum T, Díez J, Jaisser F, et al: Myocardial fibrosis: Biomedical research from bench to bedside. Eur J Heart Fail 19: 177-191, 2017.

27. Voorhees AP and Han HC: Biomechanics of cardiac function. Compr Physiol 5: 1623-1644, 2015.

28. Nielsen SH, Mouton AJ, DeLeon-Pennell KY, Genovese F, Karsdal M and Lindsey ML: Understanding cardiac extracellular matrix remodeling to develop biomarkers of myocardial infarction outcomes. Matrix Biol 75-76: 43-57, 2019.

29. Chistiakov DA, Orekhov AN and Bobryshev YV: The role of cardiac fibroblasts in post-myocardial heart tissue repair. Exp Mol Pathol 101: 231-240, 2016.

30. van Putten S, Shafieyan Y and Hinz B: Mechanical control of cardiac myofibroblasts. J Mol Cell Cardiol 93: 133-142, 2016.

31. Gabbiani G: The myofibroblast in wound healing and fibrocontractive diseases. J Pathol 200: 500-503, 2003.

32. Dobaczewski M, Chen W and Frangogiannis NG: Transforming growth factor (TGF)- $\beta$ signaling in cardiac remodeling. J Mol Cell Cardiol 51: 600-606, 2011.

33. Shinde AV, Humeres C and Frangogiannis NG: The role of $\alpha$-smooth muscle actin in fibroblast-mediated matrix contraction and remodeling. Biochim Biophys Acta Mol Basis Dis 1863: 298-309, 2017. 
34. Luo K and Lodish HF: Positive and negative regulation of type II TGF-beta receptor signal transduction by autophosphorylation on multiple serine residues. EMBO J 16: 1970-1981, 1997.

35. Li PF, He RH, Shi SB, Li R, Wang QT, Rao GT and Yang B: Modulation of miR-10a-mediated TGF- $\beta 1 /$ Smads signaling affects atrial fibrillation-induced cardiac fibrosis and cardiac fibroblast proliferation. Biosci Rep 39: BSR20181931, 2019.

36. Khalil H, Kanisicak O, Prasad V, Correll RN, Fu X, Schips T, Vagnozzi RJ, Liu R, Huynh T, Lee SJ, et al: Fibroblast-specific TGF- $\beta$-Smad $2 / 3$ signaling underlies cardiac fibrosis. J Clin Invest 127: 3770-3783, 2017.

37. Wang Y, Chu J, Yi P, Dong W, Saultz J, Wang Y, Wang H, Scoville S, Zhang J, Wu LC, et al: SMAD4 promotes TGF- $\beta$-independent NK cell homeostasis and maturation and antitumor immunity. J Clin Invest 128: 5123-5136, 2018.

38. Ding Z, Liu Y, Ruan J, Yang S, Yu H, Chen M, Zhang Y and Wang T: Bioactive constituents from the whole plants of Gentianella acuta (Michx.) Hulten. Molecules 22: E1309, 2017.

39. Feng CY, Wu Q, Yin DD, Li B, Li SS, Tang ZQ, Xu YJ and Wang LS: Determination of xanthones and flavonoids of methanol extracts obtained from different parts of the plants of three Gentianaceae species. J Pharm Biomed Anal 161: 455-463, 2018.
40. Ren K, Su H, Lv LJ, Yi LT, Gong X, Dang LS, Zhang RF and Li MH: Effects of four compounds from Gentianella acuta (Michx.) Hulten on hydrogen peroxide-induced injury in H9c2 cells. Biomed Res Int 2019: 2692970, 2019.

41. Tungmunnithum D, Thongboonyou A, Pholboon A and Yangsabai A: Flavonoids and other phenolic compounds from medicinal plants for pharmaceutical and medical aspects: An overview. Medicines (Basel) 5: E93, 2018.

42. Hammad S, Cavalcanti E, Werle J, Caruso ML, Dropmann A, Ignazzi A, Ebert MP, Dooley S and Giannelli G: Galunisertib modifies the liver fibrotic composition in the Abcb4Ko mouse model. Arch Toxicol 92: 2297-2309, 2018.

43. Luangmonkong T, Suriguga S, Adhyatmika A, Adlia A, Oosterhuis D, Suthisisang C, de Jong KP, Mutsaers HAM and Olinga P: In vitro and ex vivo anti-fibrotic effects of LY2109761, a small molecule inhibitor against TGF- $\beta$. Toxicol Appl Pharmacol 355: 127-137, 2018.

This work is licensed under a Creative Commons Attribution-NonCommercial-NoDerivatives 4.0 International (CC BY-NC-ND 4.0) License. 